\title{
La tragèdie privée. Reflexions sur Pasolini et le théâtre
}

1. Durant les premières années frioulanes Pasolini, mû par le désir d'expérimenter, passe sans cesse d'un genre à l'autre dans sa production littéraire. Certes, par la suite l'auteur continuera à alterner les genres et même les moyens expressifs, conscient comme il est des limites intrinsèques des champs explorés. Mais dans cette première période des années quarante l'utilisation pasolinienne des multiples formes littéraires n'est aucunement signe de crise, elle fait au contraire parti d'un plan minutieusement projeté. Il s'agit d'un vaste programme d' écriture autobiographique qui, se servant des genres les plus divers et utilisant comme moyens d'expression aussi bien la poésie que la prose, a pour but de nous brosser "le portrait de l'artiste jeune par lui-même." Dans son effort d'insérer sa propre expérience (à l'instant même oû il la vit) dans un récit exemplaire et strictement chronologique, Pasolini cherche à tirer profit de la poésie en dialecte ou en langue italienne, des lettres, du théâtre, des récits, des essais, et même du journal intime en vers. Il se propose de représenter l'apprentissage de l'écrivain, ainsi que le procès d'individuation par lequel il se développe comme sujet de parole, et son détachement du stade imaginaire originel, sa conquête du language, et son entrée dans le monde réel.

A l'intérieur de ce project complexe d'une écriture autobiographique des années quarante, le genre théâtral est justement utilisé par Pasolini pour représenter le moment moins subjectif, celui oû la confession personnelle trouve un auditoire, l'instant de la sortie du rêve. Dans le théâtre cela implique que la confession sexuelle, au lieu de s'exprimer dans l'espace clos du journal intime en vers, s'installe dans la réalité sociale provoquant le scandale. Nous pensons plus particulièrement à une page du roman Amado mio, oû pour "la première fois" le "secrèt" de Pasolini, le fait qu'il éprouve "de l'inclination pour les garçons," est brutalement révelé au cours d'une conversation avec une amie, et "objectivé de façon étrange par la voix de quelqu'un d'autre." Il est significatif que ce dialogue révélateur et scandaleux est justement prononcé à l'occasion de la représentation d'une pièce de théâtre écrite par Pasolini, une "fable dramatique" (inédite) intitulée $I$ fanciulli e gli elfi, dans laquelle ce même "secrèt dramatique" est dissimulé sous forme allégorique. Dans ce texte le "fanciulli" (petits garçons) sont en 
effet capturés par le "elf" (elfes) sur l'ordre d'un "ogre" méchant et cannibale (et son rôle est joué par Pasolini même); ils deviennent cependant eux-mêmes à leur tour les "séducteurs" de leurs bourreaux jusqu'au moment oû "tous ensemble s'enfuient" vers le monde de la normalité. L'ogre reste seul, "réduit au désespoir et au ridicule," tandisque "les bons et les convertis s'en vont chantant." Cette fable nous révèle-même si présentée comme négative et tachée d'un sentiment de culpabilité-la vérité sur la sexualité pasolinienne; cette sexualité que quelques années plus tard on appellera "corruption."

Hegel déja soulignait le rapport privilégié qui existe entre le genre drammatique et son publique, le "devoir" envers ce publique, que l'auteur dramatique s'engage à remplir, la nécessité "instantanée" d'un texte drammatique écrit pour être représenté sans qu'il se renferme en une consommation toute personnelle et intériorisée. C'est précisément ce rapport avec le public qui est indiqué, selon nous, dans I Turcs tal Friul écrit au printemps de l'année 1944. Ici il ne s'agit plus de petites expériences dialoguées, à tendance hermétique, mais d'une structure drammatique traditionnelle qui a non seulement pour sujet des rapports interpersonnels, mais qui s'ouvre en outre sur une écoute réelle de la part d'un public. La nécessité de la communication ne coïncide pas automatiquement avec la volonté de transmettre un message. Dans I Turcs tal Friul il n'y a aucun nouveau message par rapport à ceux déjà apparus dans les textes pasoliniens. Cette histoire située à Casarsa vers la fin du quinzième siècle lors d'une invasion turque, cette attente désespérée de la mort et de la destruction, tandis que les paysans se sont réunis pour prier et pleurer, cette série d'annonces toujours plus inquiétantes et menaçantes (incendies, morts, pillages) qui se termine de façon tout à fait imprévue par un miracle-la tempête éloigne l'envahisseur-tout cela n'est qu'un passage en revue des vieux motifs. Cet ouvrage est une récapitulation non seulement des matériaux frioulans, mais encore de tous les thèmes et de l'iconographie de L'usignolo della Chiesa Cattolica. L'unique nouveauté réside tout au plus dans une forme inédite de métaphore politique, par laquelle on dénonce, justement dans ces mois drammatiques, le nazisme par envahisseur turcs interposés. Le théâtre devient alors un mode d'expression privilégié non seulement quant à son rapport avec le public, mais aussi comme première tentative de communication historique et politique. Le dialecte utilisé dans I Turcs tal Friul est très différent de celui des autres ouvrages frioulans. Il n'est plus question d'un régistre "pre-linguistique," la métaphore d'un absence de communication. Le dialecte devient au contraire (adopté par le théâtre et implicitement rapproché de la tradition populaire du théâtre dialectal) le moyen de la communication la plus complète. 
2. Si maintenant nous nous transportons au coeur des années soixante, nous assistons, parallélement au rapport avec le grand public que le cinéma impose au metteur en scène Pasolini, à un retour de l'intérêt porté au théâtre. Mais il ne s'agit plus d'une recherche de syntonie avec le public; et ces ouvrages drammatiques ne s'insèrent pas aisément dans le reste de l'oeuvre pasolinien de la même période. Au beau milieu d'une époque de sa vie de nouveau réaliste et engagée (nous pensons aux moyens audio-visuel utilisés comme documentaire, mais aussi à la poésie comme dénonciation de l'horreur, dans un lien renouvelé entre l'auteur et son' public) Pasolini semble encore se contredire, indiquer un autre voie, une nouveauté ou (peut-être) au contraire un retour nostalgique. Violent mouvement de rébellion contre l'univers bourgeois, le théâtre pasolinien de ces années-là voudrait indiquer in extremis une issue à l'ordre capitaliste, ainsi qu'à cette poésie illuminée qui (bien que pour la dénoncer) s'y accomode. Ce théâtre est le soubresaut d'un auteur qui se refuse d'accepter la dimension réaliste de l'écriture, aussi bien qu'une poésie entièrement mêlée à l'horreur quotidien. Instrument de contestation, sans compromis vis à vis du présent, le théâtre pasolinien prend presque automatiquement la forme de la tragédie, genre extrêmement problématique dans la littérature du vingtième siècle.

S'il est vrai que le tragique refuse, comme disait Lukacs, "l'anarchie du clair-obscur" en faveur d'une clarté paradoxale qu'on ne saurait confondre avec l'ambiguité ou l'incertitude; s'il est vrai que le tragique consiste en un pari pascalien qui fixe ses choix et se prononce pour un Dieu qui se cache éternellement: alors nour pouvons affirmer que dans la littérature italienne de l'après-guerre Affabulazione (la première tragédie de Pasolini) constitue un rare example d'un heureux réemploi de structures de la tragédie ancienne, mêlée à et revécue tout au long d'une écriture poétique moderne. Affabulazione nous montre, comme l'indique le titre, l'effort de raconter encore quelque-chose dans un monde qui n'admet plus de choses ni de paroles nouvelles: c'est l'utilisation de la forme tragique à fin de dépasser cette société de la consommation à laquelle le, recueil poétique Poesia in forma di rosa s'était abandonnée. Ce mouvement tragique a, en tant que négation de la norme sociale, une résonance monstrueuse et paradoxalement exemplaire dans ce monde mesquin du présent. Un tel défi scandaleux (homosexualité, inceste, violence et mort) rend en quelque sorte reconnaissable l'Unicum, permet d'afficher la différence totale à l'intérieur de cet univers de l'uniformité totale. Le geste tragique repropose le miracle de l'unicité dans ce monde d'homogénéité et d'équivalences: miracle finalement reconnaissable et exemplaire.

Le geste tragique (un père qui tue son fils) réalisé dans Affabulazione 
reste cependant une exception dans le théâtre pasolinien des années soixante. Toutes ses autres tragédies ne font plus que mettre en scène l'impossibilité et la dégradation du tragique et marquent en même temps le retour inexorable de son théâtre à cette dimension tout intériorisée et lirique de l'autobiographie, dimension que dans ses pièce des années quarante il avait justement tenté de fuire. D'ailleurs, cette trańsformation de la scène théâtrale en scène personnelle, ce genre d'exercice de solipsisme se rencontre déjà dans des textes plus anciens que le tragédies des années soixante. Nous pensons à la Storia interiore encore inédite, composée aux environs de 1960 et remaniée sous le titre $\mathrm{Nel}$ ' 46 . Comme le double titre révele déjà, il s'agit d'une énième évocation de journal intime, mais sous forme drammatique cette fois. Le drame fonctionne alors comme confession, l'histoire personnelle de Pasolini devient matière de spectacle, prend la structure du dialogue, mais non plus pour être présentée à un public attentif, ou pour instaurer une dialectique avec le monde extérieur (comme dans I Turcs tal Friul), mais pour se perdre dans l'introspection, dans le débat de l'âme avec lui-même. Cette solitude du narrateur qui se raconte assume déjà les structures et la tension de la tragédie, mais d'une tragédie privée, écrite pour soi-même et donc paradoxalement dépourvue de toute charge rituelle ou exemplaire.

Dans Pilade, la pièce écrite à la même époque que Affabulazione (et dont la belle mise en scène de Mario Feliciano a si bien mis en évidence l'organisation profonde), le caractère autobiographique du protagoniste est encore accentué. Dans cette pièce nous n'assistons pas, comme à la fin de Affabulazione, à un geste de négation totale, mais plutôt à une fuite ("une fuite par laquelle je veux me libérer"); à une renonciation et a une sorte de compensation plus qu'à la tentative de nous imposer avec violence le scandale tragique. A la différence du père dans Affabulazione, Pilade et Electre ne nous imposent rien, ne constituent pas un exemple, mais s'isolent dans leur ghetto. Au dehors rien ne change, et la transgression est reléguée à l'ombre (déjà ici vaguement ridicule) d'un lieu clos. L'anormalité qui "contredit tout" n'est assurément pas quelque-chose "dont tous, sans exception, se scandaliseront," comme le pense Electre; c'est plutôt la pauvre illusion de deux êtres qui se croient tragiques, tandis qu'au fond il ne sont que deux personnes pleurnichardes et à l'esprit élégiaque. Ici, aucun Dieu, aucun miracle comme dans Affabulazione, le scandale ne se situe plus dans la realité mais dans une illusion reconstruite dans l'intimité du rêve. Ce n'est pas un hasard si ce texte se termine par une interrogation au sujet du tragique, et de la faillite de son geste. Aucune nouveauté donc, et aucune tragédie. Le refus final que Pilade oppose à la Raison, en réfutant point par point la dialectique d'Athéne réapparue, n'est pas une "protestation," 
mais seulement un "rêve" dans lequel il est vrai que "l'on n'a plus besoin d'être consolé," mais qui constitue déjà en lui une autre forme de consolation. Pilade peut s'écrier: "Tu sois maudite, Raison," mais il n'exprime pas la vérité quand il dit qu'ainsi sa vie deviendra une chose "tragique et ridicule." Pour les protragonistes des textes théâtrals suivants il ne restera, hélas, que le ridicule.

3. L'énonciation théâtrale se réfère pourtant toujours au public dans sa fonction de destinataire. Dans n'importe quel genre de théâtre le destinataire a un poids plus considérable que dans tout autre forme de communication littéraire. C'est cette notion centrale que semble animer le "Manifesto per un nuovo teatro," paru en 1968 sur la revue Nuovi Argomenti. Deux chapitres de l'écrit s'intitulent en effect "Qui seront les destinataires du nouveau théâtre" et "Destinataires et spectateurs." Pasolini se propose donc de trouver pour son théâtre un public différent de celui traditionnel. Le résultat est pourtant plutôt étrange:

L'auteur d'un texte théâtral n'écrira plus pour ce public qui, par definition, a toujours été le public théâtral. ... Les destinataires du nouveau théâtre ne seront ni divertis ni scandalisés par ce nouveau théâtre, parce que, appartenant aux groupes les plus avancés de la bourgeoisie, ils seront en tout les égaux de l'auteur des textes.

Si on lit entre le lignes de cette affirmation, la remarque est pour ainsi dire scandaleuse: le théâtre nouveau "a pour destinataire les mêmes groupes par lesquels il est produit." Dans le "Manifesto" Pasolini théorise en somme un théâtre qui a son auteur pour tout public. L'idée du groupe avancé peut en effect facilement être réduit au point de ne recouvrir que le groupe qui se constitue d'un seul component: l'auteur de ce que Pasolini nomme le "théâtre de la parole." Si les auteurs deviennent le public, le public disparîat et avec lui la notion originelle du théâtre comme manifestation et rite socials, du théâtre comme exemple. Tout en feignant de chercher un public, Pasolini nie en réalité l'existence $d u$ public tout court, en supprimant chaque distance et en ramenant tout â lui-même en tant que producteur-destinataire. Ce qui reste est la parole, comprise comme énonciation d'une thèse, comme verbalisation d'une "idée." Et ce n'est pas un hasard si Pasolini a écrit ses textes aprês la lecture des dialogues de Platon, écrits dans lesquels le silence et l'absoluité tragiques transparaissent à travers la parole pédagogique et le débat dialectique:

Le théâtre de la Parole cherche son "espace théâtral" non pas dans le monde environnant, mais à l'intérieur de la tête. Du point de vue technique un tel "espace théâtral" se présentera de front: texte et acteurs face à face avec le public. L'absolue égalité culturelle entre ces deux interlocuteurs, qui se regardent droit 
dans le yeux, garantie une réelle démocratie, aussi scénique.

La tête du public devient la tête de l'auteur, et dans ce ... tête à tête il nous semble retrouver la figure connue du sujet dialoguant avec lui même devant un miroir. Le texte, se déroulant à l'interieur de la "tête," retourne aussi à sa source, ou pour mieux dire ne s'en éloigne jamais.

Ce théâtre non théâtral ne permet, en théorie, aucune forme de mise en scène. C'est pourquoi Pasolini peut même affirmer la liquidation de Brecht ("il a été le dernier homme de théâtre qui a pu faire une révolution théâtrale à l'intérieur du théâtre même"); justement parce que le "théâtre de la parole" est encore en un sens brechtien, critique, réflexif, distancié, mais en même temps étranger à toute hypothèse de spectacle réel. Pasolini assume effectivement le théâtre tragique comme modèle sublime et parfait contre le présent dégradé, contre le "théâtre du bavardage," mais en même temps il réduit son théâtre au mutisme scénique, et l'abaisse avec un plaisir sadique assez ambigu au niveau de la survivance culturelle, de l'activité exclusivement littéraire. La mise en scène, la récitation, la mimique, tout devient secondaire par rapport au Texte, conçu regoureusement comme série verbale. Tout disparaît littéralement devant la parole:

Une des caractéristiques fondamentales du "théâtre de la parole" sera l'absence pour ainsi dire complète d'action scénique ... la disparition presque totale de la mise en scère.

Qui mieux que l'auteur purrait assumer en même temps les fonctions de la scène et de l'acteur? Il est le moyen parfaitement transparent à travers qui, et pour ainsi dire à partir de qui et vers qui, peut retentir la parole sans être méconnue ni occulté. Ce théâtre refuse toute existence à un texte non verbal, à un code gestuel par example; il refuse en effet les conditions minimales indispensables à l'existence du théâtre. Comme la poésie le théâtre renvoit à l'écrit, et ceci avec une violente réduction des phénomènes scéniques au profit de la trame écrite et des répliques du dialogue. Si le texte dramatique peut être en général considéré comme le dépôt (ècrit) d'une manifestation scénique qui à l'origine était une destruction de l'ordre social, dans ce cas le théâtre pasolinien, qui se réduit entièrement au texte, est le contraire d'une transgression sociale: c'est un texte poétique que l'on peut peut-être déclamer ou lire lors d'une lecture publique, mais ce n'est absolument pas une mise en scène rituelle. Comme dit Pasolini même, c'est un théâtre de poésie, un moyen indirect pour écrire de vers: une écriture personnelle, le plaisir de la solitude.

4. Après le moment de la grande espérance tragique avec Affabulazione, le "Manifesto" inaugure donc une réorganisation réductive du théâtre. On 
y voit justement formulé sur le plan théorique l'échec du geste tragique que nous avions déjà observé à la fin de Pilade: le théâtre devient luimême l'emblème de ce ghetto qui enfermait Pilade et Electre. Le but plus authentique que Pasolini se propose dans ses autres oeuvres théâtrales est précisément la mise en scène de cette désillusion du tragique et cette misère du théâtre, sa fonction d'illusionnisme privé pour un auteur solitaire. Nous pensons à Orgia, texte contemporain au "Manifesto" et pour ainsi dire une application officielle de ses principes théoriques. Nous pensons également au ridicule et à l'impuissance qui caractérisent Porcile et surtout Calderón.

Dans la dernière pièce de théâtre de Pasolini, Bestia da stile, nous touchons au dernier stade de cette dégradation du tragique. Dans un geste provocateur le tragique devient ici une série de confessions liriques et de monologues encore plus compacts que dans le textes précédents. Et si dans sa préface Pasolini liquide toute la scène italienne en dénonçant la qualité inferieure de ses "textes écrits," c'est justement pour classer son propre teste comme appartenant à la poésie, à la littérature pure. "Autobiographie" sarait alors le terme parfait pour indiquer Bestia da stile. Plus encore qu'ailleurs le théâtre devient le contraire du théâtre: réconstruction du temps passé sur l'ancien modèle déjà experimenté par Pasolini dans ses années frioulanes. Et si les premiers vers de Bestia da stile déclarent sous forme d'acrostiche: "Viva lo stile," cela implique que le théâtre adopte encore une fois la langue de la littérature et que tout se transforme en une voix de poète qui s'élève solitaire pour parler de soi. Le théâtre ne se réduit pas seulement à l'autobiographie, mais devient éloge de la lirique pure.

Dans Bestia da stile le ton est pourtant vaguement ironique. Pasolini répète l'expérience autobiographique frioulane, mais il en sourit. Si autrefois raconter sa propre histoire signifiait la revivre au moment même de la parole tout en feignant toujours un distance, maintenant, après tant d'années, la distance est mélancholiquement réelle et bien visible. Pasolini adopte donc une stylisation toute proche de l'autocaricature. L'autobiographie devient ridicule: non plus l'enchantement de la perfection mais la désillusion, la fin. L'ambiguité persiste, mais comme affreuse impossibilité d'atteindre l'exemplarité. Le visage sublime de la poésie n'est donc qu'un masque, une tromperie, qui peut aller jusqu'à la bouf fonnerie. Ecrire des vers devient un indécent collage de textes d'autrui, un mélange de citations tirées d'essais our même para-littéraires. Si le théâtre est mort, la poésie par contre devient théâtre, mais un théâtre encore une fois vidé de son essence et de motivation. Derrière l'Idée de la Poésie il y a cette frivole vacuité à laquelle arrive à la limite le Pasolini des années soixante-dix: le 
vide.

\section{Université de Groningen}

(traduit de l'italien par Michèle Rinaldi-Bonte)

\section{OEUVRES CONSULTÉES}

Pasolini, Pier Paolo. Amado mio précedé d'Atti impuri. Éd. C. D’Angeli. Milan:

Garzanti, 1982. . I Turcs tal Friul. Éd. L. Ciceri. Udine: Forum Julii, 1976.

Affabulazione. Pilade. Milan: Garzanti, 1977.

Calderón. Milan: Garzanti, 1973.

. Porcile. Orgia. Bestia da stile. Milan: Garzanti, 1976.

. "Manifesto per un nuovo teatro." Nuovi Argomenti (gennaio-marzo, 1968).

Rinaldi, R. Pier Paolo Pasolini. Milan: Mursia, 1982. 